\title{
Correction to: On the Deligne-Lusztig involution for character sheaves
}

\section{Alexander Yom Din ${ }^{1}$}

Published online: 31 October 2020

(c) Springer Nature Switzerland AG 2020

\section{Correction to: Selecta Mathematica (2019) 25:49 https://doi.org/10.1007/s00029-019-0495-6}

I am grateful to D. Gaitsgory for pointing out the mistake.

Claim 3.1 should be stated as follows:

Claim 3.1 Let $\mathcal{C}, \mathcal{D} \in \operatorname{Lin}_{k}$ be compactly generated categories, and let $F: \mathcal{C} \rightarrow \mathcal{D}$ be such that $F^{R}$ and $F^{R R}$ exist and are continuous, as well as $F^{L}$ and $F^{L L}$ exist (in other words, both $F$ and $F^{\vee}$ should admit a proper right adjoint). Then the following diagram commutes:

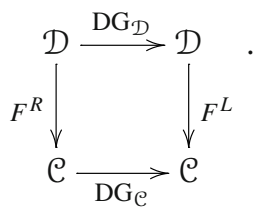

This correction, of demanding $F^{R R}$ and $F^{L L}$ to exist (and be continuous) is because we need $F^{L}$ and $\left(F^{R}\right)^{\vee}$ which appear in Diagram (3.1) to commute with limits, so that the functors in the diagram between the opposite categories will commute with colimits, guaranteeing that the squares in the diagram commute (as one can check that the squares commute on compact objects, and then one wants to extend by continuity).

Claim 3.2 should be stated as follows:

Claim 3.2 Let $\mathcal{C} \in \operatorname{Lin}_{k}$ be a compactly generated category. Suppose that $\operatorname{Id}_{\mathcal{C}} \in$ $\operatorname{Hom}(\mathcal{C}, \mathcal{C})$ is reflexive. If $\mathrm{DG}_{\mathcal{C}}$ admits a proper right adjoint then it is right-invertible. In particular, if $\mathrm{DG}_{\mathcal{C}}$ and $\mathrm{DG}_{\mathcal{C}} \vee$ admit proper right adjoints, then $\mathrm{DG}_{\mathcal{C}}$ is invertible.

The original article can be found online at https://doi.org/10.1007/s00029-019-0495-6.

\footnotetext{
$凶$ Alexander Yom Din ayomdin@gmail.com

1 Pasadena, CA, USA
} 
This correction is because we use the bottom square of Diagram (3.1) in the proof, and therefore, as explained above, we need the right adjoint not only exist and be continuous, but also itself to have a continuous right adjoint.

Corollary 3.3 should be stated as follows:

Claim 3.3 Let $X$ be a space. If $\mathrm{DG}_{D(X)}$ admits a proper right adjoint, then $\mathrm{DG}_{D(X)}$ is invertible.

This correction is clear, as this corollary is proved by applying Claim 3.2.

The proof of Proposition 5.1 can be written: Apply Claim 3.1 to $F=$ pres $_{I}$ (taking into account Theorem 4.2 and Corollary 4.3).

The proof of Proposition $\mathbf{5 . 5}$ should be written: By Corollary 3.3, it is enough to show that $\mathrm{DG}_{D(G \backslash G)}$ admits a proper right adjoint. This follows from Theorem 5.2, since by Corollary 4.3 the functor $\operatorname{pind}_{I}^{-}$and $\operatorname{pres}_{I}$ admit all iterated adjoints.

Publisher's Note Springer Nature remains neutral with regard to jurisdictional claims in published maps and institutional affiliations. 